ORIGINAL ARTICLE

\title{
The implications of NICE guidelines on the management of children presenting with head injury
}

\section{J Dunning, J P Daly, R Malhotra, P Stratford-Smith, J-P Lomas, F Lecky, J Batchelor, $K$ Mackway-Jones, for the Children's Head injury Algorithm for the Identification of significant Clinical Events Study (CHALICE Study)}

Arch Dis Child 2004;89:763-767. doi: 10.1136/adc.2003.042523

See end of article for authors' affiliations

Correspondence to: MrJ Dunning, Research Fellow, Emergency Medicine Research Group, Department of Emergency Medicine, Manchester Royal Infirmary, Oxford Road, Manchester M13 9WL, UK; joeldunning@ doctors.org.uk

Accepted

19 February 2004
Background: NICE guidelines for the management of head injury were published in June 2003. Their recommendations differ markedly from previous guidelines published by the Royal College of Surgeons (RCS). In place of skull radiography and admission, computed tomography (CT) is advocated. The impact of these guidelines on service provision in the UK is unknown.

Methods: Data on all clinical correlates of children presenting with any severity of head injury was collected in three hospitals in the northwest of England. The current skull radiograph (SXR), CT scan, and admission rates were determined. The rates of SXR, CT scan, and admission that should have occurred when following either the RCS or NICE guidelines were then determined.

Results: Data from 10965 patients who attended three hospitals between February 2000 and August 2002 was studied. Twenty five per cent of patients received a SXR, $0.9 \%$ a CT scan, and $3.7 \%$ were admitted. Strict adherence to the RCS guidelines would have resulted in a 50\% SXR rate, a $1.6 \%$ CT scan rate, and a $7.1 \%$ admission rate. Adherence to NICE guidelines would result in a $0.3 \%$ SXR rate, an $8.7 \%$ CT scan rate, and a $1.4 \%$ admission rate, although the CT rate would drop to $6.3 \%$ if vomiting three or more times in the under $12 \mathrm{~s}$ was used instead of more than one vomit.

Conclusions: The new NICE guidelines do not increase the workload caused by patients attending with head injury but they move their management from the observation ward to the radiology department.
O ne million patients with head injuries attend emergency departments each year in the UK, of which up to $50 \%$ are children..$^{1-3}$ In contrast to the high incidence of head injury, the mortality rate is comparatively low (6-10 per 100000$)$, and as few as $0.2 \%$ of all people attending the emergency department suffer a fatal outcome. $^{45}$

Thus although emergency clinicians see a large number of patients with minor head injury, they rarely see patients who then suffer life threatening intracranial complications. Therefore they must rely on clinical guidelines to govern the management of these patients.

Over the past decade a number of decision rules have been developed in order to identify high risk patients in the minor head injury category who require a CT scan. ${ }^{6-11}$ UK practice has consistently been centred on guidelines based on high rates of skull radiography and admission, and low rates of computed tomography (CT). The most recent, widely adopted version of these guidelines was published in June 1999 by the Royal College of Surgeons of England. ${ }^{12}$

The National Institute of Clinical Excellence (NICE) has recently published a markedly different protocol for the management of head injuries in adults and children for widespread use in the UK. ${ }^{13}$ Based on the Canadian Head CT rule, ${ }^{6}$ these guidelines advocate a management strategy based on early computed tomography for high risk patients and a marked reduction in skull radiography and admission.

The implication of these guidelines on UK hospital resources in terms of increased CT requests and possibly lower admission rates are currently unknown. A comprehensive database of 11000 consecutive children presenting with a head injury was therefore analysed to investigate the impact of these new guidelines.

\section{METHODS}

\section{Study type}

This was a subanalysis of a larger multicentre prospective cohort study currently underway in the northwest of England.

\section{Patient selection}

A prospective cohort study of all children presenting to 10 hospitals in the northwest of England with any severity of head injury, was conducted between February 2000 and August 2002. This study analysed all patients in this database who attended Alder Hey Hospital, Stepping Hill Hospital, or Manchester Royal Infirmary, during this time period.

\section{Inclusion criteria}

All patients under 16 years of age were included. Any patient presenting to the emergency department with a head injury of any severity was included in the study. Of note, there was no necessity for loss of consciousness or amnesia for inclusion into our study. There were no exclusion criteria other than refusal to enter the study by the patient or guardian.

\section{Data collection}

A specifically designed proforma was created for data collection. This proforma collected data on over 40 clinical correlates pertaining to the head injury, including correlates on the mechanism of injury, symptoms, signs, and management of the patient. Every physician who participated in the

Abbreviations: CT, computed tomography; GCS, Glasgow Coma Score; NICE, National Institute of Clinical Excellence; RCS, Royal College of Surgeons; SXR, skull radiograph 
study was given a one hour training session on the study and the use of this proforma for data collection. Response rates and quality of completion was monitored in all centres on a monthly basis in order to ensure high compliance. Importantly, all physicians were asked to follow the 1999 Royal College of Surgeons guidelines for the management of head injuries; the guidelines were printed clearly on the front of every proforma.

\section{Outcome measures}

Three outcome measurements were analysed, skull radiograph (SXR), head CT, and need for admission. The actual management of the patient was taken from proforma documentation. Any proforma documenting a SXR, admission or CT scan, or with data missing from any of these categories, had this data verified by consulting hospital records. Some management decisions depend on the presence of a skull fracture. All SXRs were checked by radiologists and diagnosis of a fracture was determined only by the radiologist's report.

Management of these patients according to the Royal College of Surgeons guidelines and the NICE guidelines were determined for each individual patient in the study and this was compared to actual management.

\section{Statistical analysis}

Descriptive analysis was conducted using SPSS version 11.5. In order to provide clinicians with an estimate of the variability in the frequency that investigations might be required on a weekly basis, confidence intervals for a theoretical group of 100 patients attending the emergency department with head injury were constructed using a Monte-Carlo simulation. ${ }^{14}$ One hundred patients were randomly selected from our dataset and the number of investigations required was calculated. Their data were replaced and the process was repeated 1000 times. The 95\% confidence intervals of this dataset are presented. The simulation was performed using STATA version 7.

\section{Ethics}

Multicentre ethical approval was obtained for this study and consent to participate in this study obtained from all patients or guardians prior to entry to the study.

\section{RESULTS}

A total of 10965 patients entered this study from Alder Hey, Stepping Hill, and Manchester Royal Infirmary. Table 1 shows their demographics.

Of the 10965 patients, attending physicians requested 2746 skull radiographs (25\%). Only 94 CT scans were requested $(0.9 \%)$ and 409 patients were admitted $(3.7 \%)$. Another 511 patients were observed in the emergency department for over three hours $(4.7 \%)$, although 477 of these patients went home within six hours.

When the Royal College of Surgeons guidelines were strictly applied to the database it was found that these guidelines dictate that 5519 (50.3\%) of patients should have had a SXR. Figure 1 shows the reasons for a SXR request by these guidelines. It can be seen that the majority of SXRs were required by RCS guidelines due to the presence of any bruising or swelling. It is clear that physicians are not using the presence of any bruise or swelling to request a SXR as the actual number of SXRs requested is far fewer than that predicted. The figure for SXRs required by the RCS guidelines was recalculated for an increasing size of bruise or swelling. If a bruise or swelling over $4 \mathrm{~cm}$ was used instead of any bruise or swelling, $3122(28 \%)$ of patients would have required a SXR. So it seems that physicians may be regarding bruise or swellings only over $4 \mathrm{~cm}$ as significant.
Table 1 Patient demographics

\begin{tabular}{|c|c|c|}
\hline \multirow[b]{2}{*}{ Patient demographics } & \multicolumn{2}{|c|}{ Patients (total $n=10965$ ) } \\
\hline & $\mathbf{n}$ & $\%$ \\
\hline \multicolumn{3}{|l|}{ Sex } \\
\hline Male & 7070 & $64.5 \%$ \\
\hline Female & 3831 & $34.9 \%$ \\
\hline Missing & 64 & $0.6 \%$ \\
\hline \multicolumn{3}{|l|}{ Age } \\
\hline $0-6$ months & 454 & $4.1 \%$ \\
\hline 6-12 months & 773 & $7.0 \%$ \\
\hline $1-2$ years & 1822 & $16.6 \%$ \\
\hline $2-5$ years & 3037 & $27.7 \%$ \\
\hline $5-12$ years & 3245 & $29.6 \%$ \\
\hline $12-16$ years & 1561 & $14.2 \%$ \\
\hline Missing & 73 & $0.7 \%$ \\
\hline \multicolumn{3}{|l|}{ Mechanism } \\
\hline Fall from height & 2143 & $19.5 \%$ \\
\hline Slip, trip, or fall & 5809 & $53.0 \%$ \\
\hline Projectile or object & 1137 & $10.4 \%$ \\
\hline Assault & 476 & $4.3 \%$ \\
\hline Collision mechanism & 792 & $7.2 \%$ \\
\hline RTA-occupant & 77 & $0.3 \%$ \\
\hline RTA-cyclist & 373 & $3.4 \%$ \\
\hline RTA-pedestrian & 132 & $1.2 \%$ \\
\hline Missing & 26 & $0.2 \%$ \\
\hline \multicolumn{3}{|l|}{ Symptoms } \\
\hline Loss of consciousness & 521 & $4.8 \%$ \\
\hline Amnesia & 314 & $2.8 \%$ \\
\hline Vomiting & 1185 & $10.8 \%$ \\
\hline Headache & 2246 & $20.5 \%$ \\
\hline GCS 15 & 10700 & $97.6 \%$ \\
\hline
\end{tabular}

Applying RCS guidelines to the dataset, 171 CT scans would have been required ( $1.6 \%)$, most of these being due to a skull fracture being found on SXR. Full application of the RCS guidelines would have required 775 admissions (7.1\%).

The NICE guidelines were applied to the dataset (full results are given in table 2 and NICE guidelines for a CT are given in table 3 ). There are very few reasons for requesting a SXR under the NICE guidelines and we found that only 30 SXRs should have been performed due to suspicion of nonaccidental injury. Following exactly the recommendations for a CT scan by NICE it was found that 957 (8.7\%) CT scans would have to have been required in our dataset. Figure 2 gives the reasons for requiring a CT scan. It was found that by far the most common reason for requesting a CT scan was the

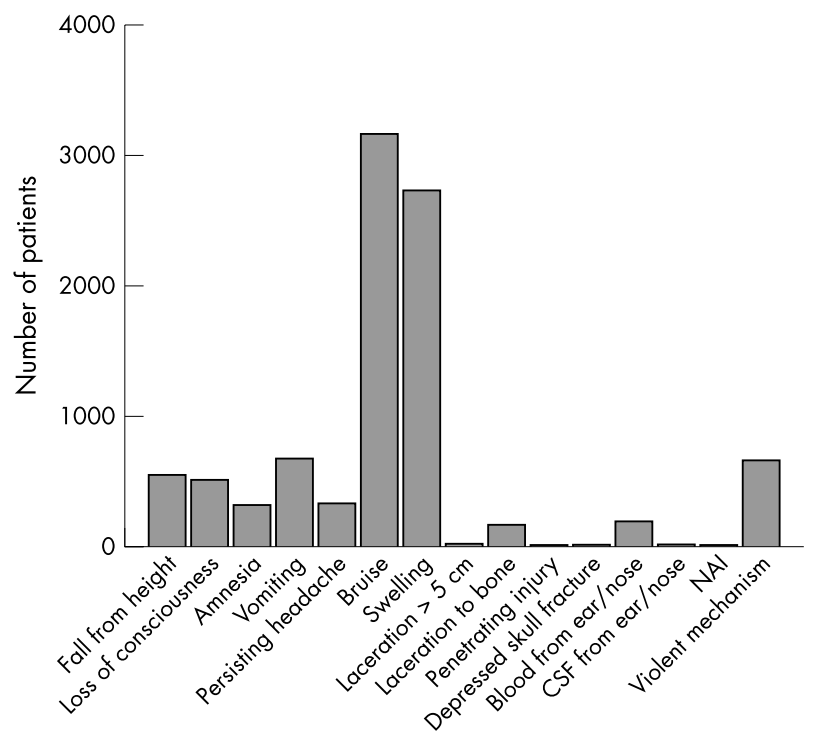

Figure 1 SXR required by RCS guidelines 1999. 


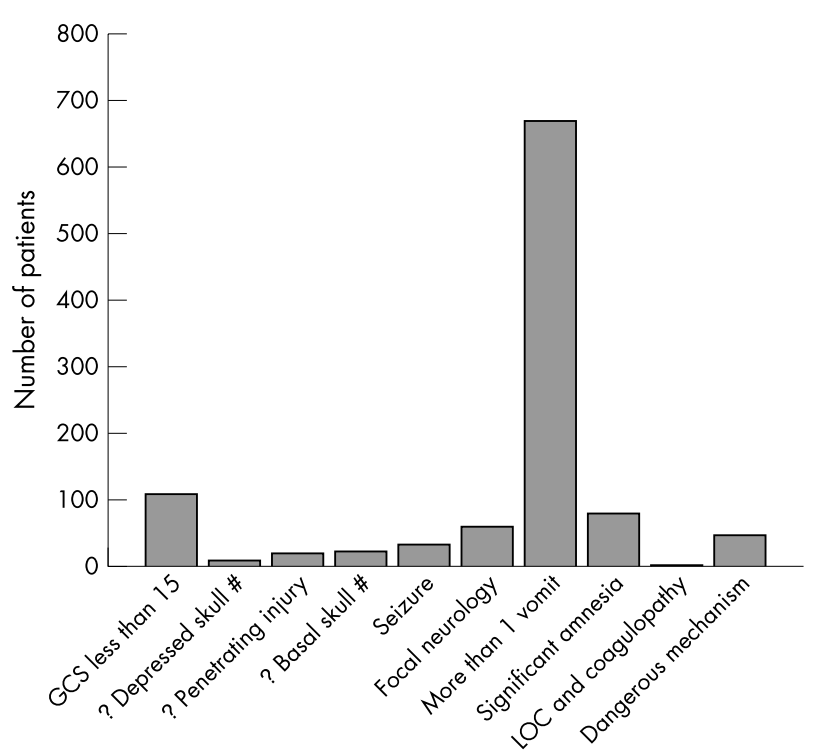

Figure 2 Reasons for a CT under NICE guidelines. \#, fracture; ?, query.

presence of more than one vomit. There is a caveat in the NICE guidelines for vomiting in the under $12 \mathrm{~s}$, allowing clinicians to use their clinical judgement as to whether more than one vomit is a sufficient reason to request a CT scan. We therefore recalculated these figures for our database using three or more vomits as the number of vomits required to request a CT scan in the under 12s. The number of CT scans now required by NICE dropped to 687 (6.3\%).

The number of admissions required under NICE guidelines was calculated to be 151 (1.4\%), of which 107 were due to an admission GCS score of less than 15. These patients would be eligible for discharge on returning to GCS 15 under NICE guidelines, after a CT scan.

Our database contains only information on children and adolescents. In order to gain an insight as to the possible impact that the NICE guidelines would have on an adult population, we analysed only those patients between 12 and 16 years of age. Table 4 presents the full results; it was found that the rates of investigation and management are similar to the full database, although the CT scan rate as determined by NICE was a little higher at $10.7 \%$. It can be seen by the current management rates of admission and SXR that the adolescents perhaps contain fewer trivial injuries, which may account for the higher predicted CT scan rate.

There has been some concern that the new NICE head injury guidelines identify a higher proportion of patients at high risk compared to the Royal College of Surgeons guidelines. We therefore looked at the number of patients that each guideline assigned as high risk. A high risk patient was defined as any patient that qualified for either a CT scan or an admission. The Royal College of Surgeons guidelines allocated 787 patients as high risk (7.2\%), whereas the NICE guidelines allocated 983 patients (8.9\%). However, if the higher threshold of vomiting was used for the NICE guidelines in patients under 12 , only 714 patients would have been allocated as high risk (6.5\%). Therefore NICE guidelines do not allocate a higher proportion of patients as high risk compared to RCS guidelines.

In order to provide clinicians with an estimate of the upper limits of investigation that might be required in their department, we modelled a theoretical cohort of 100 patients and calculated the $95 \%$ confidence intervals for each management option. Table 5 presents the full results. It was found that the upper limit of the number of CT scans that might be required for a group of 100 patients attending the emergency department using the NICE guidelines was $11.1 \%$.

\section{DISCUSSION}

NICE guidelines aim to unify clinical practice in the UK for the management of head injuries around a single, best evidence guideline. However, in order to implement these practice parameters, clinicians must not only have confidence in the methodology and research background of the guidelines, but also an understanding as to the likely impact on service provision. NICE guidelines have been produced to the highest methodological standards, but were unable to provide an accurate indication of the likely impact that these guidelines would have in terms of rates of imaging and admission. We have shown that admission rates are currently $3.7 \%$ of all head injury attendances and that this rate is likely to drop to $1.4 \%$ with full application of the guidelines. In its place the CT scan rate will rise to between $6 \%$ and $8 \%$ depending on how clinicians apply the vomiting recommendation.

Clinicians managing service provision not only require an insight into the mean number of investigations required by the NICE guidelines but also the likely upper limits. A theoretical cohort of 100 patients was modelled in order to obtain clinically relevant confidence intervals. This number was chosen as it represents about one month of head injury attendances in a medium sized emergency department. It was found that using an interpretation of the vomiting criterion of three or more, while the mean CT rate was $6.3 \%$ the upper limit of CT scan requests can be expected to be around $11 \%$ of all emergency department attendances.

We have shown that a large number of the requested CT scans will be performed due to the presence of vomiting. Furthermore, we have shown that the way in which this criterion is interpreted by clinicians will be of paramount importance in the number of scans performed.

Our cohort of patients is in the childhood population only. Although the NICE guidelines are the same for adults and children, children are more likely to vomit than adults.

Table 2 Management of 10965 patients with head injury.

\begin{tabular}{llll}
\hline & Skull radiograph & CT scan & Hospital admission \\
\hline Actual management, \% (SE) & $25 \%(0.41 \%)$ & $0.86 \%(0.088 \%)$ & $3.7 \%(0.18 \%)$ \\
Royal College of Surgeons & $n=2746$ & $n=94$ & $n=409$ \\
guidelines, \% (SE) & $50 \%(0.48 \%)$ & $1.6 \%(0.12 \%)$ & $7.1 \%(0.24 \%)$ \\
NICE guidelines, \% (SE) & $n=5519$ & $n=171$ & $n=775$ \\
NICE guidelines with 3 or more & $0.27 \%(0.049 \%)$ & $8.7 \%(0.27 \%)$ & $1.4 \%(0.11 \%)$ \\
vomits in the under 12s as the criteria & & $n=957$ & $n=151$ \\
for vomiting, \% (SE) & & $6.2 \%( \pm 0.23 \%)$ & \\
\hline
\end{tabular}


Table 3 NICE guidelines for CT scanning of high risk patients

Patients who have sustained a head injury and present with any one of the following risk factors should have $\mathrm{CT}$ scanning of the head immediately requested:

- GCS less than 13 at any point since the injury

- GCS equal to 13 or 14 at 2 hours after the injury

- Suspected open or depressed skull fracture

- Any sign of basal skull fracture (haemotympanum, "panda" eyes, cerebrospinal fluid otorrhoea, Battle's sign)

- Post-traumatic seizure

- Focal neurological deficit

- More than one episode of vomiting (clinical judgement should be used regarding the cause of vomiting in those aged 12 years or younger, and whether imaging is necessary)

- Amnesia for greater than 30 minutes of events before impact. The assessment of amnesia will not be possible in pre-verbal children and is unlikely to be possible in any child aged under 5 years

$\mathrm{CT}$ should also be immediately requested in patients with any of the following risk factors, provided they have experienced some loss of consciousness or amnesia since the injury:

- Age greater than or equal to 65 years

- Coagulopathy (history of bleeding, clotting disorder, current treatment with warfarin)

- Dangerous mechanism of injury (a pedestrian struck by a motor vehicle, an occupant ejected from a motor vehicle, or a fall from a height of greater than 1 metre or five stairs). A lower threshold for height of falls should be used when dealing with infants and young children (that is, aged under 5 years)

Therefore in order to try and provide some indication as to the likely CT scan rates in adults, we performed a subanalysis with only the patients from 12-16 years of age in our database. We found a $10.7 \%$ CT scan rate, which is similar to the scan rate for all children. Although adolescents have a lower incidence of vomiting and therefore may be expected to have a lower CT rate, there is a higher rate of very minor head injuries in the very young. This may account for our finding that the CT rate was similar for adolescents and younger patients. We propose that it is likely that our findings in adolescents may be extrapolated to young and middle aged adults, although the major confounding factor is likely to be alcohol consumption. The intoxication rate in our adolescent age group was less than $1 \%$, whereas in young adult populations this rate may be as high as $50 \%$. However, the NICE guidelines make no specific recommendations regarding presence of intoxication, and patients will qualify for a CT scan only when the level of intoxication is enough to reduce their GCS.

A weakness of our paper is that the figures for management dictated by the NICE guidelines were assessed retrospectively for our database rather than prospectively looking at how physicians apply the guidelines. We can therefore not comment on how physicians will apply the NICE guidelines. We predict however that while the CT guidelines are likely to be closely followed, physicians may request a CT scan in vomiting patients less often than recommended, and may either observe or admit these patients, as physicians may be uncomfortable discharging a vomiting patient even with a normal CT scan.

To our knowledge, there have been no other studies modelling the impact of the NICE guidelines in paediatric head injuries. Our findings of current practice are similar to those of other cohort studies looking at all severities of head injuries in children in the UK. Lloyd and colleagues ${ }^{15}$ reported a cohort of 9269 childhood head injuries from 1993 to 1995 in the UK. They found a SXR rate of $64 \%$ and an admission rate of $9.1 \%$. Their CT scan rate was $1.7 \%$, with skull fracture being the most common reason for a CT scan.

Nee and colleagues ${ }^{16}$ reported a cohort of 5416 children and adults with head injury and found an incidence of $12 \%$ for vomiting in children, of which $3.6 \%$ were multiple vomits. A study of 12395 patients that had sustained a head injury in Chester (published 1978) found that $7 \%$ of all patients had vomited. ${ }^{17}$ Boulis and colleagues ${ }^{18}$ reported a cohort of 1000 children with head injury having a SXR at the Royal Free Hospital and found an incidence of $17 \%$ for vomiting, $7 \%$ loss of consciousness, and 9\% headache. They reported that the most common reason for requesting a SXR was soft tissue injury to the scalp.

We have also found a marked difference between actual management and the guidelines that the clinicians had been asked to follow. Strict adherence to the RCS guidelines would have resulted in a 50\% SXR rate, a $1.6 \%$ CT scan rate, and a $7.1 \%$ admission rate. In clinical practice we found that the rate was half this for each category, at $25 \%, 0.9 \%$ and $3.7 \%$ respectively. Our findings that clinicians were not closely following the RCS guidelines are in agreement with a previous study that found a $47 \%$ non-adherence to guidelines for SXRs when the use of the Harrogate criteria was assessed in $1990 .^{19}$

Finally, our finding that the NICE guidelines do not actually increase the number of patients that are being labelled as "high risk" is interesting. Taking the liberal interpretation of vomiting, the RCS guidelines allocated 7.2\% of all patients as high risk, whereas the NICE guidelines allocate $6.5 \%$ as high risk. Therefore the effect of the NICE guidelines is not to increase the workload of clinicians caring for head injured patients but to move this workload from the observation ward to the radiology department.

\section{Conclusion}

The NICE guidelines will result in a CT scan rate of $6-9 \%$ for all children attending the emergency department with head

Table 4 Management of patients 12-16 years of age $(n=1561)$

\begin{tabular}{llll}
\hline & Skull radiograph & CT scan & Hospital admission \\
\hline Actual management, \% (SE) & $27.9 \%(1.1 \%)$ & $1.2 \%(0.28 \%)$ & $5.3 \%(0.57 \%)$ \\
& $n=436$ & $n=19$ & $n=83$ \\
Royal College of Surgeons & $56.7 \%(1.3 \%)$ & $1.6 \%(0.3 \%)$ & $7.8 \%(0.68 \%)$ \\
guidelines, \% (SE) & $n=885$ & $n=25$ & $n=122$ \\
NICE guidelines, \% (SE) & $0.2 \%(0.11 \%)$ & $10.7 \%(0.78 \%)$ & $1.9 \%(0.28 \%)$ \\
& $n=3$ & $n=167$ & $n=19$ \\
\hline
\end{tabular}


Table $595 \% \mathrm{Cl}$ for rates of imaging or management that might be expected for 100 patients attending the emergency department with a head injury (modelled by MonteCarlo simulation)

\begin{tabular}{llll}
\hline & Skull radiograph & CT scan & Hospital admission \\
\hline Actual management & $16.8-33.2 \%$ & $0-2.7 \%$ & $0.13-7.4 \%$ \\
Royal College of Surgeons guidelines & $40.6-60.3 \%$ & $0-3.9 \%$ & $1.9-12.0 \%$ \\
NICE guidelines & $0-1.3 \%$ & $3.2-14.2 \%$ & $0-3.8 \%$ \\
NICE guidelines with 3 or more vomits & & $1.5-11.1 \%$ & \\
in the under 12s as the criteria for & & & \\
vomiting & & \\
\hline
\end{tabular}

injury, with an upper limit to this figure not exceeding $14 \%$. The interpretation of the severity of vomiting will have a great impact on this figure, but overall the NICE guidelines do not increase the number of patients allocated as high risk; they merely move their treatment from the ward to the radiology department.

\section{Authors' affiliations}

J Dunning, P S Smith, Manchester Royal Infirmary, UK

J P Daly, R Malhotra, J-P Lomas, Manchester University, UK

J Batchelor, K Mackway-Jones, Emergency Medicine at the Manchester Royal Infirmary, UK

F Lecky, The Trauma Audit and Research Network at Hope Hospital, University of Manchester, UK

Grant support: The Emergency Medicine Research Group is grateful for the support given by the Enid Linder Research Fellowship from the Royal College of Surgeons of England, and a grant from the Children's Brain Injury Trust

The research was performed at the Emergency Medicine Research group, Department of Accident and Emergency Medicine, Manchester Royal Infirmary, Oxford Road, Manchester M13 9WL, UK

\section{REFERENCES}

1 Teasdale GM. Head injury. J Neurol Neurosurg Psychiatry 1995:58:526-39.

2 Jennett B, MacMillan R. Epidemiology of head injury. BMJ 1981;282:101-4.

3 Brookes M, MacMillan R, Cully $S$, et al. Head injuries in accident and emergency departments. How different are children from adults? J Epidemiol Community Health 1990;44:147-51.

4 Swann IJ, MacMillan R, Strong I. Head injuries at an inner city accident and emergency department. Injury 1981;12:274-8.
5 Swann IJ, Teasdale GM. Current concepts in the management of patients with so-called 'minor' or mild' head injury. Trauma 1999;1:143-5.

6 Stiell IG, Wells GA, Vandemheen K, et al. The Canadian CT Head Rule for patients with minor head injury. Lancet 2001;357:1391-6.

7 Borczuk P. Predictors of intracranial injury in patients with mild head trauma. Ann Emerg Med 1995;25:731-6.

8 Servadei F, Teasdale G, Merry G. Defining acute mild head injury in adults: a proposal based on prognostic factors, diagnosis, and management. Journal of Neurotrauma 2001;18:657-64.

9 Miller EC, Holmes JF, Derlet RW. Utilizing clinical factors to reduce head CT scan ordering for minor head trauma patients. J Emerg Med 1997;15:453-7.

10 Haydel MJ, Preston CA, Mills TJ, et al. Indications for computed tomography in patients with minor head injury. N Engl J Med 2000;343:100-5.

11 The EAST Practice Management Guidelines Work Group. Practise management guidelines for the management of mild traumatic brain injury. The Eastern Association for the Surgery of Trauma, 2001.

12 Royal College of Surgeons of England. A working party on the management of the head injured patient. Holborn, London: RCS England, Lincoln's Inn Fields, 1999

13 The National Collaborative Centre for Acute Care. Head injury: triage, assessment, investigation and early management of head injury in infants, children and adults. Clinical Guideline N0234. Published by National Institute of Clinical Excellence. http://www.nice.org.uk, 2003.

14 Efron B, Tibshirami R. An introduction to the bootstrap. London: Chapman and Hall, 1993 (reprinted 1998).

15 Lloyd DA, Carty H, Patterson M, et al. Predictive value of skull radiography for intracranial injury in children with blunt head injury. Lancet 1997;349:821-4

16 Nee PA, Hadfield JM, Yates DW, et al. Significance of vomiting after head injury. J Neurol Neurosurg Psychiatry 1999;66:470-3.

17 Gorman DF. The utility of post-traumatic skull X-rays. Arch Emerg Med 1987:4:141-50.

18 Boulis ZF, Dick R, Barnes NR. Head injuries in children-aetiology, symptoms, physical findings and x-ray wastage. BrJ Radiol 1978;51:851-4.

19 de Lacey G, McCabe M, Constant O, et al. Testing a policy for skull radiography (and admission) following mild head injury. Br J Radiol 1990;63:14-18.

\section{Making Health Care Safer 2004}

21-22 October 2004

Royal College of Physicians, London

A two day conference for all professionals dedicated to providing safer health care for all.

Register now! Early booking discount available.

See website for details: www.quality.bmipg.com 